\title{
Pacemaker Dysfunction due to a Large Th rombus on Ventricular Lead
}

\author{
Zhen Xia, ${ }^{1}$ Jianhua Yu, ${ }^{1}$ Juxiang Li, ${ }^{1}$ Qinmei Xiong, ${ }^{1}$ Yanhua Tang, ${ }^{2}$ Sujuan Yan, ${ }^{1}$ Kui Hong, ${ }^{1}$ \\ Xiaoshu Cheng ${ }^{1}$ \\ Departments of ${ }^{1}$ Cardiology and ${ }^{2}$ Cardiac Surgery, The Second Affiliated Hospital of Nanchang University, Nanchang, China
}

\section{ABSTRACT}

Background: Pacemaker lead-related thrombosis is a rare but severe complication in patients with pacing lead implantation in the right ventricle. We present a case with recurrent syncope after single-chamber implantable cardioverter defibrillator (ICD) implantation. Pacing lead-related thrombosis was observed during open-heart surgery. This induced intermittent pacemaker dysfunction and recurrent syncope.

Case Presentation: A 67-year-old male patient presented with frequent episodes of syncope and a history of dilated cardiomyopathy and paroxysmal ventricular tachycardia. Normal coronary angiography was found, and therefore a single-chamber ICD was implanted into the right ventricle to prevent cardiac events in 2013. However, he was referred to our hospital because of recurrent syncope 3 to 4 years after ICD implantation. A comprehensive investigation was performed to find out the etiology for the recurrent syncope. Pacing lead thrombosis was finally observed during openheart surgery, which can introduce intermittent pacemaker dysfunction. After the thrombus was removed and the lead was separated from the posterior leaflet of the tricuspid valve, the ICD functioned normally after reprogramming. Oral anticoagulant was prescribed after discharging. During the 1-year follow-up period, this patient was free of syncope.

Conclusions: This case illustrated that pacemaker leadassociated thrombosis should be considered when the cardiac implantable electronic device fails to prevent patients from having cardiac events. Oral anticoagulant might be important for preventing thrombosis among patients with ICD implantation into the right ventricle.

\section{BACKGROUND}

The implantable cardioverter defibrillator (ICD) has been reported to carry a significant risk of complications, including

Received November 9, 2018; received in revised form Fanuary 1, 2019; accepted fanuary 3, 2019.

Z.X. and F.Y. contributed equally to this study.

Correspondence: Fuxiang Li, No.1 Minde Road, Donghu District, Nanchang City, 330006, P.R. China; +86-13657092311; fax: +86-791-86297276 (e-mail:ljx912@126.com). lead displacement, pneumothorax, hematoma, and infection. [Ezzat 2015] Pacemaker lead-related thrombosis is a rare but severe complication. Implanting pacing and defibrillator leads is usually straightforward and is generally associated with a low risk of thromboembolic events. In addition, there is no need for routine anticoagulant therapy. Therefore, it has been widely performed in single-chamber ICD implantation.

We report a rare case of lead-related thrombosis after ICD implantation, which induced intermittent pacemaker dysfunction and recurrent syncope. After removal of the thrombi during open-heart surgery, the pacemaker function returned to normal when it was fully programmed.
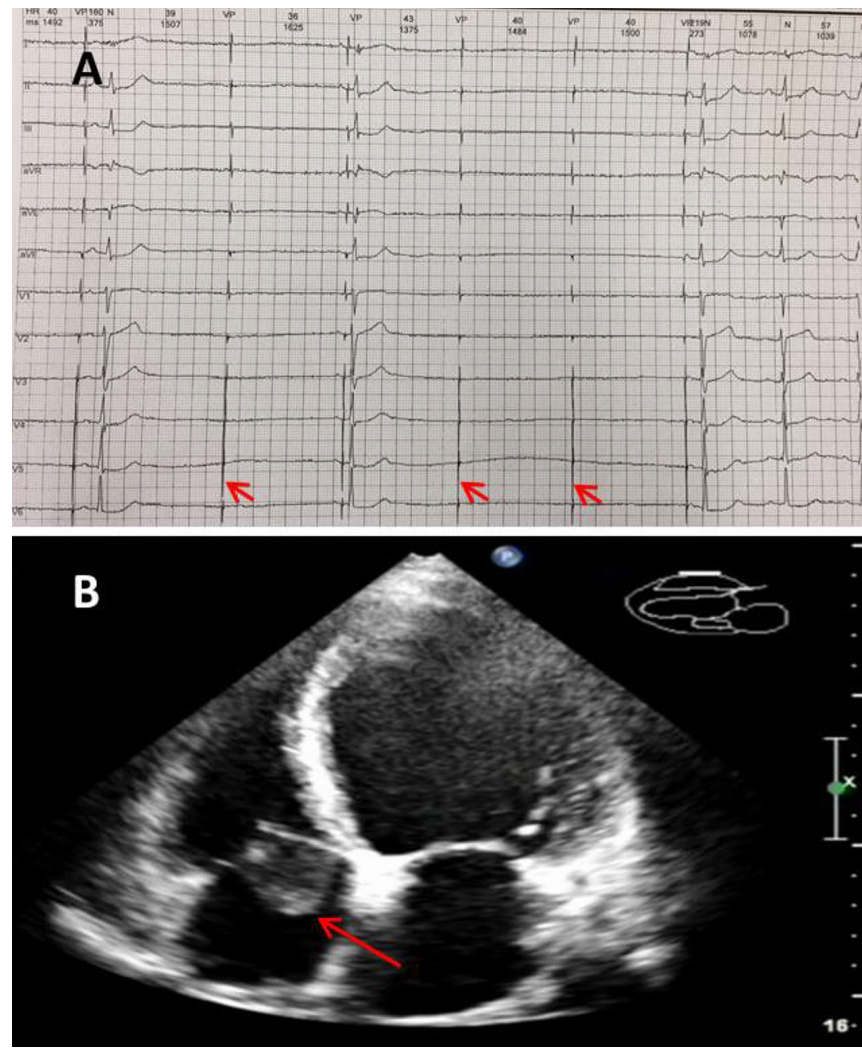

(A), Pacemaker dysfunction. The red arrow represents absent QRS wave after the pacing signal, indicating poor cardiac pacing. (B), The huge mass in the atrium shown in echocardiography. The red arrow represents the echogenic mass swinging through the tricuspid valve between the right atrium and ventricle during the cardiac cycle. 


\section{CASE PRESENTATION}

A 67-year-old male patient presented with frequent episodes of syncope and a history of dilated cardiomyopathy and paroxysmal ventricular tachycardia. Normal coronary angiography was found, and therefore a single-chamber ICD was implanted into the right ventricle to prevent sudden cardiac death in 2013. However, he was referred to our hospital because of recurrent syncope 3 to 4 years after ICD implantation.

Sinus bradycardia, poor cardiac pacing, and intermittent long intervals of 4.36 seconds were observed in the ambulatory electrocardiogram monitoring (Figure, A). When we programmed the pacemaker parameters, they were $5.0 \mathrm{~V}$ for threshold, $1.6 \mathrm{mV}$ for sensing, $48 \Omega$ for defibrillation coil impedance. The battery capacity of ICD was still sufficient. There was no episode of ventricular tachycardia and ventricular fibrillation in previous records. Transesophageal echocardiography at admission revealed a slightly echogenic mass swinging through the tricuspid valve between the right atrium and ventricle during the cardiac cycle, which indicated a probability of thrombus (Figure, B). No abnormality was found in detection of inflammatory markers, including white blood cell counts, C-reactive protein level. D-dimer and coagulation parameters were in the normal range. No fever, diarrhea, cough, or expectoration was present.

Clinical decision was made upon a comprehensive examination. A complication of lead-related thrombosis but not infective endocarditis was considered. Anticoagulation therapy using low-molecular-weight heparin and bridging warfarin was carried out for 15 days. However, subsequent echocardiography showed similar to the previous observation at admission.

On the basis of the episode of syncope, the high risk for pulmonary embolism, and the pacemaker's dysfunction with high pacing threshold and low perception, open-heart surgery was recommended to extract the pacing electrode. After obtaining signed informed consent, pacing electrode extraction and thrombectomy were performed under general anesthesia. The extraction was done during cardiopulmonary bypass with aortic cross-clamping. On visual inspection during the procedure, the pacemaker lead appeared encapsulated with adhesion of an old thrombus at the superior vena cava-right atrium junction. Thrombi were detached and removed from the adherent posterior leaflet. Histopathologic examination confirmed the aseptic thrombus. The ICD was left in place because the detection of parameters was identified to be appropriate during the procedure (Table). Warfarin treatment was continued after discharge. During a 3-month follow-up period, the patient was free of syncope episode, and the parameters of the programmed-controlled pacemaker remained appropriate.

\section{DISCUSSION AND CONCLUSIONS}

Pacemaker lead-related thrombosis is a rare complication of pacemaker implantation, occurring in $0.6 \%-3.5 \%$ of cases in previous studies [Barakat 2000; D'Aloia 2013]. No anticoagulant therapy is recommended for patients with ICD
Programmed Parameters Detected before and after Cardiac Surgery

\begin{tabular}{lcc}
\hline & Preoperative & Postoperative \\
\hline Threshold & $5.0 \mathrm{~V}$ & $<0.25 \mathrm{~V}$ \\
Perception & $1.6 \mathrm{mV}$ & $5.6 \mathrm{mV}$ \\
Impedance & $300 \Omega$ & $460 \Omega$ \\
Defibrillation impedance & $48 \Omega$ & $46 \Omega$
\end{tabular}

implantation. The thrombi were potentially secondary to the endothelial injury by long-term pacemaker presence [Do Carmo Da Costa 2002; D'Aloia 2013]. Platelet aggregation after pacemaker implantation might be another explanation for the formation of thrombosis [Palatianos 1994]. Additionally, hypercoagulable states should also be taken into account, including heart failure, neoplasia, pregnancy, etc.

Buttigieg et al and Raut et al previously reported successful thrombectomy and pacemaker lead extraction in patients with atrial thrombosis documented by echocardiography [Buttigieg 2015; Raut 2015]. However, there are rare reports on pacing dysfunction caused by the thrombus on the ventricular lead. In the present case, adhesion of the thrombus to the tricuspid valve may have contributed to the changes of pacemaker parameters, ie, high threshold and low perception. During cardiac systole the thrombus will flow to the atrial side, and the pacemaker electrode might be pulled, which can cause a slight dislocation of the electrode. Therefore, thrombus removal might be the best treatment.

In conclusion, this case illustrated that the pacemaker leadassociated thrombosis should be considered when the cardiac implantable electronic device fails to prevent patients from having cardiac events. Oral anticoagulant might be important for preventing thrombosis among patients with ICD implantation in the right ventricle.

\section{ACKNOWLEDGMENTS AND DECLARATIONS}

Funding: This work was supported by the National Natural Science Foundation of China (grant no. 81760065) and the Natural Science Foundation of Jiangxi Province (no. 2016BAB215238).

Authors' contributions: J.L. conceived and designed this case report. Z.X. and J.Y. performed the data collection and wrote the paper. Y.T. performed the cardiac surgery. Q.X. and S.Y edited the manuscript. K.H. and X.C. reviewed the manuscript. All authors have contributed to this work.

Ethics approval and consent to participate: This case report was approved by the Ethic Committee of the Second Affiliated Hospital of Nanchang University. The patient provided his verbal informed consent.

Consent for publication: Informed consent for publication was obtained from this patient.

Availability of data and materials: All data generated or analyzed during this study are included in this published article. 
Competing interests: None of the authors have any competing interests.

\section{REFERENCES}

Barakat K, Robinson NM, Spurrell RA. 2000. Transvenous pacing leadinduced thrombosis: a series of cases with a review of the literature. Cardiology. 93(3):142-8.

Buttigieg J, Asciak R, Azzopardi CM. 2015. Pacemaker lead-associated thrombosis in cardiac resynchronisation therapy. BMJ Case Rep 2015:bcr2015210314.

D'Aloia A, Bonadei I, Vizzardi E, Curnis A. 2013. Right giant atrial thrombosis and pulmonary embolism complicating pacemaker leads.
BMJ Case Rep 2013:bcr2012008017.

Do Carmo Da Costa SS, Neto AS, Costa R, Caldas JG, Filho MM. 2002. Incidence and risk factors of upper extremity deep vein lesions after permanent transvenous pacemaker implant: a 6-month follow-up prospective study. Pacing Clin Electrophysiol 25(9):1301-6.

Ezzat VA, Lee V, Ahsan S, et al. 2015. A systematic review of ICD complications in randomised controlled trials versus registries: is our 'realworld' data an underestimation? Open Heart. 2(1):e000198.

Palatianos GM, Dewanjee MK, Panoutsopoulos G, Kapadvanjwala M, Novak S, Sfakianakis GN. 1994. Comparative thrombogenicity of pacemaker leads. Pacing Clin Electrophysiol 17(2):141-5.

Raut MS, Maheshwari A, Dubey S. 2015. Thrombus on pacemaker lead. Indian Heart J 67(suppl 3):S120-1. 